\title{
玉米叶向值的全基因组关联分析
}

\author{
彭 勃 $1,{ }^{* *}$ 赵晓雷 $1,{ }^{* *}$ 王 奕 ${ }^{1, * *}$ 袁文娅 $^{1}$ 李春辉 $^{2}$ 李永祥 $^{2}$ \\ 张登峰 $^{2}$ 石云素 ${ }^{2}$ 宋燕春 ${ }^{2}$ 王天宇 ${ }^{2, *}$ 黎 裕 ${ }^{2, *}$
}

${ }^{1}$ 天津市农作物研究所 / 天津市农作物遗传育种重点实验室, 天津 $300384 ;{ }^{2}$ 中国农业科学院作物科学研究所, 北京 100081

摘 要: 叶向值是反映叶片“直”和“立”两个特性的综合指标。叶向值高的品种, 叶片直而不弯, 叶夹角小, 有利于群 体通风透光, 在群体密度较高时比平展型更容易获得高产。阐明叶向值的遗传基础, 对玉米理想株型分子设计育种具 有重要的意义。本研究以 285 份多样性玉米自交系为材料, 利用 Illumina 的 maizeSNP50 芯片基因分型结合连续 2 年 的叶向值表型鉴定, 通过全基因组关联分析方法挖掘玉米叶向值显著关联的 SNP 位点。方差分析表明, 不同自交系 的叶向值差异达到极显著水平 $(P<0.01)$ 。在最优模型选择时, 发现 $\mathrm{Q}+\mathrm{K}$ 模型最适合本研究的叶向值关联分析。在 2 个年份下, 共检测到 15 个与叶向值显著关联的位点 $(P<4.05 \mathrm{E}-5)$, 包括 27 个 SNP, 解释 $5.54 \% \sim 8.73 \%$ 的表型变异, 并 挖掘了 15 个候选基因。其中 $1.07 \mathrm{bin}$ 上的位点 2 是本研究发现的重要位点, 其候选基因可能是编码细胞周期蛋白依 赖性蛋白激酶的 Zm00001d032050，有待进一步图位克隆工作验证。

关键词: 玉米; 叶向值; 叶夹角; 单核苷酸多态性; 关联分析

\section{Genome-wide association studies of leaf orientation value in maize}

\author{
PENG Bo ${ }^{1, * *}$, ZHAO Xiao-Lei ${ }^{1, * *}$, WANG Yi ${ }^{1, * *}$, YUAN Wen-Ya ${ }^{1}$, LI Chun-Hui ${ }^{2}$, LI Yong-Xiang ${ }^{2}$, ZHANG \\ Deng-Feng ${ }^{2}$, SHI Yun-Su ${ }^{2}$, SONG Yan-Chun ${ }^{2}$, WANG Tian-Yu ${ }^{2, *}$, and LI Yu ${ }^{2, *}$ \\ ${ }^{1}$ Tianjin Crop Research Institute / Tianjin Key Laboratory of Crop Genetics and Breeding, Tianjin 300384, China; ${ }^{2}$ Institute of Crop Sciences, \\ Chinese Academy of Agricultural Sciences, Beijing 100081, China
}

\begin{abstract}
Leaf orientation value is a comprehensive index reflecting the two characteristics of "straight" and "vertical" of leaves. The varieties with high leaf orientation value have straight and not curved leaves, and small angle, which are conducive to the wind ventilation and light transmission for maize population. When the planting density is high, it is easier to obtain high yield than the expanded plant-type. It is of great significance for molecular design breeding of ideal plant type to clarify the genetic basis of leaf orientation value. In this study, 285 diverse lines genotyped by the MaizeSNP50 chip were evaluated for leaf orientation in 2017 and 2018. The genome-wide association analysis (GWAS) were used to identified the SNPs, which were significant association with leaf orientation values. The analysis of variance showed that the significant variations were observed for leaf orientation value of different inbred lines $(P<0.01)$. In the selection of the optimal model, it was found that the $\mathrm{Q}+\mathrm{K}$ model was the most suitable for the leaf orientation association analysis in this study. A total of 15 loci $(P<4.05 \mathrm{E}-5)$ were detected by GWAS, including 27 SNPs, explaining $5.54 \%-8.73 \%$ of phenotypic variation, and 15 candidate genes were mined in two years. Among them, site 2 in 1.07 bin was an important site found in this study, and its candidate gene might be Zm00001d032050 encoding cyclin dependent protein kinase, which needed to be further confirmed by map-based cloning.
\end{abstract}

Keywords: maize; leaf orientation value; leaf angle; single nucleotide polymorphism; association analysis

\footnotetext{
本研究由国家自然科学基金项目 (31601308), 青年科研人员创新研究与实验项目 (2018004), 天津市自然科学基金项目 (19JCZDJC34500)和天津市农业科学院院长基金项目(17007)资助。

This study was supported by the National Natural Science Foundation of China (31601308), the Innovative Research and Experiment Project for Young Researchers (2018004), the Tianjin Natural Science Foundation (19JCZDJC34500), and the President's Fund of Tianjin Academy of Agricultural Sciences (17007).

*通信作者(Corresponding authors): 王天宇, E-mail: wangtianyu@caas.cn; 黎裕, E-mail: 1iyu03@caas.cn

** 同等贡献(Contributed equally to this work)

第一作者联系方式: 彭勃, E-mail: snbopeng@163.com
}

Received (收稿日期): 2019-12-03; Accepted (接受日期): 2020-01-15; Published online (网络出版日期): 2020-02-17.

URL: http://kns.cnki.net/kcms/detail/11.1809.S.20200215.2228.006.html 
过去的几十年中, 玉米株型结构的改良, 尤其 是直立叶型的品种对玉米产量的遗传增益起了重要 的作用 ${ }^{[1-2]}$ 。直立叶型品种比平展叶型品种通常更耐 密植, 通过较高的叶面积指数来增加群体光能截获, 促进群体光合作用, 进而获得高产 ${ }^{[1,3-4]}$ 。叶向值是可 以同时反映叶片“直”和“立”两个特性的综合指标。 穗上叶向值高, 上部叶片直而不弯, 叶夹角小而上 冲, 是理想株型育种的重要选择目标 ${ }^{[3]}$ 。因此, 阐明 玉米叶向值的遗传基础对玉米理想株型育种具有重 要的意义。

玉米叶向值是典型的数量性状, 受多基因控制, 遗 传基础复杂 ${ }^{[5-6]}$ 。前人以不同杂交组合构建的 $F_{2} 、 F_{2: 3}$ 、 四交 $F_{1}$ 单株、RILs群体为材料, 利用SSR分子标记和 连锁作图方法，在染色体的很多区段都检测到了控 制叶向值的QTL ${ }^{[5-11]}$ 。其中, 主效QTL(解释表型变异 率大于 $10.00 \%$ 的QTL)遗传效应大，受环境影响小， 历来是分子育种和QTL图位克隆优先关注的位点 [2,12-13]。在 $1.01 \mathrm{bin} 、 1.02 \mathrm{bin} 、 1.10 \mathrm{bin} 、 2.02 \mathrm{bin} 、$ 2.04 bin 、 3.06 bin 、 3.08 bin 、 3.09 bin、 4.01 4.02 bin、 $7.00 \mathrm{bin}$ 和 $7.04 \mathrm{bin}$ 上先后发现了叶向值的主效 $\mathrm{QTL}^{[5-11]}$ 。随着技术的发展, 近年来, 利用第3代分子 标记SNP标记通过连锁作图方法, 在3.05 bin、7.05 bin 上也发现了叶向值的主效QTL ${ }^{[14-15]}$ 。Zhao等 ${ }^{[6]}$ 整合了 前人关于叶夹角、叶向值、叶长、叶宽、叶面积和 叶长/叶宽6个叶型性状的QTL定位结果, 通过元分析 方法, 发现 22 个meta-QTL (mQTL), 并预测了其候选 基因。

综上, 连锁作图为玉米叶向值遗传基础的解析 提供了重要的参考, 但是由于受到群体重组率、标 记密度等限制, QTL定位区间较大, 而且仅仅能分析 少数几个等位基因。与连锁分析互补, 全基因组关 联分析 (genome-wide association study, GWAS)可 以高效挖掘多个多样性玉米种质资源的优异等位 基因。关联分析已经被用于叶夹角遗传基础的剖 析 ${ }^{[12,16-17]}$, 而且 $Z m C L A 4^{[12]} 、 U P A 1$ 和 $U P A 2^{[2]}$ 、 $Z m I L I 1^{[13]} 4$ 个控制叶夹角的QTL已经实现了图位克 隆。在玉米叶向值上, 仅 $\mathrm{Lu}$ 等 ${ }^{[18]}$ 以 80 份玉米骨干自 交系为材料, 利用 $1.49 \times 10^{6}$ 个 SNP结合 2 年的表型鉴 定, 通过全基因组关联分析(GWAS)方法检测到 33 个与叶夹角和叶向值显著相关的 SNP $(P<1.00 \mathrm{E}-6)$, 并在显著的SNP位点上下游 $9.7 \mathrm{~kb}$ 的LD衰减距离内, 挖掘到 22 个叶夹角候选基因和 7 个叶向值候选基因。 相比于叶夹角, 叶向值全基因组关联分析的报道较
少。本研究以285份遗传多样性丰富的玉米自交系 为关联群体, 结合覆盖玉米整个基因组的 56,110个 SNP标记，通过全基因组关联分析的方法(GWAS) 挖掘玉米种质资源中调控叶向值的基因资源, 旨在 为耐密植的玉米理想株型分子设计育种提供理论 参考。

\section{1 材料与方法}

\section{1 材料}

所用材料由中国农业科学院作物科学研究所 提供, 是从 890 份包含玉米自交系核心种质 ${ }^{[19]}$ 和近 年来我国玉米育种常用优良自交系，以及近1000份 其他国家的自交系 ${ }^{[20]}$ 中，选择出适应天津地区种 植, 且遗传多样性较高的285份玉米自交系, 其中 271 份来源于我国北方、黄淮海、西南主要玉米生 产区域, 12份来源于美国, 1份来源于非洲, 1份来源 于墨西哥。

\section{2 方法}

\subsection{1田间试验设计全部材料于2017年和2018} 年连续2年种植在天津市农业科学院武清创新基地, 采用完全随机区组试验设计, 单行区, 2次重复, 行 长 $3 \mathrm{~m}$, 行距 $0.6 \mathrm{~m}$, 密度 67,500 株 $\mathrm{hm}^{-2}$ 。施肥、灌溉、 防虫和除草同当地大田管理。

在玉米散粉后 $10 \mathrm{~d}$, 从第 4 株开始, 每行连续 测量 5 株穗上 3 片叶的叶夹角 $(\theta)$ 、叶长 $(\mathrm{L})$ 和高点长 (叶片基部至叶片最高点的距离, Lf), 并采用Pepper 等 $^{[21]}$ 提出的方法计算叶向值 (leaf orientation value, $\mathrm{LOV}), \mathrm{LOV}=1 / n \Sigma(90-\theta) \times(\mathrm{Lf} / \mathrm{L}), n$ 表示测定叶 片数。

\subsection{2 表型数据分析 利用SPSS 22.0统计软件} 对 2 个环境下叶向值(LOV)的平均值、标准差、频率 分布等进行描述性统计分析。利用R语言 (http://www. r-project.org/) 1me4包的混合线性模型, 计算每份材 料最佳线性无偏预测(best linear unbiased prediction, BLUP)值，模型中将基因型(Geno)、年份(Year)、基 因型与年份互作 (Geno: Year) 和重复 (Rep) 都作为随 机效应, 所得到的每份材料的BLUP值与截距相加, 即可得到每份材料真正的遗传效应表型数值, 用于 后续的全基因组关联分析。

\subsection{3 基因型鉴定与 SNP质量控制 285份材料} 利用MaizeSNP50 BeadChip芯片基因分型, 该芯片 包括 56,110 个SNP标记, 基因型数据由中国农业科 学院作物科学研究所提供。利用PLINK1.09软件 ${ }^{[22]}$ 
过滤掉最小等位基因频率（maf $<0.05$, 基因型缺失 率 (geno) $>0.2$ 的SNP标记。最终 39,827 个 SNP通过软 件质量控制。

\subsection{4 连锁不平衡(linkage disequilibrium, LD)分析}

利用Haploview 4.2软件计算SNP之间的LD衰减 距离，同一染色体上两个位点之间的距离以 $25 \mathrm{~kb}$ 的 基本分类单元分类，并计算不同分类单元的平均 $\mathrm{LD}^{[23]}$ 。标记间相关系数 $\left(r^{2}\right)$ 小于 0.1 , 认为没有相关 性 ${ }^{[24]}$ 。

\subsection{5 全基因组关联分析 (1)國值确定：由于很} 多SNP之间可能存在高度连锁不平衡, 因此采用 GEC 软 件 (http://grass.cgs.hku.hk/gec/estimateB.php? function=Bonferroni) ${ }^{[25]}$ 计算有效标记数 (effective number, $\mathrm{Ne})$ ，并根据软件计算出的建议阈值 $(1 / \mathrm{Ne})$ 作为叶向值与 SNP间是否显著关联的依据。(2)模型 选择与全基因组关联分析：利用TASSEL5.0软件的3 种常用的模型, 即只控制群体结构的Q模型 $(\mathrm{Q})$ 、只 控制亲缘关系的 $K$ 模型 $(K)$ 、控制群体结构和亲缘关 系的 $\mathrm{Q}+\mathrm{K}$ 模型 $(\mathrm{Q}+\mathrm{K})$ 对叶向值进行全基因组关联分 析, 通过比较QQ图来选择最优模型, 并分析最优模 型下SNP与叶向值的关联。(3)候选基因预测: 定义 同一染色体上物理距离小于连锁不平衡(LD)衰减距 离的所有显著关联的SNP为 1 个位点, 在每个位点内 $-\lg (P)$ 峰值的 SNP上下游各LD衰减距离的区间范围 内挖掘候选基因 ${ }^{[26]}$ 。基于参考基因组(B73_RefGen $\mathrm{v} 4)$, 在ensembl网站上搜索每个位点内的所有候选 基因, 利用 ensembl数据库和Gene Ontology数据库 预测候选基因执行的生物学功能, KEGG数据库预 测候选基因可能参与的生物学通路信息。此外, 通 过序列比对水稻(RAP数据库)或者拟南芥(Tair数据 库)相关同源基因的功能来注释玉米候选基因。结合 上述基因注释以及 MaizeGDB上qTeller的表达部位 分析, 在每个QTL区间内确定 1 个最有可能的叶向值 候选基因。

\section{2 结果与分析}

\section{1 叶向值表型数据分析}

不同年份叶向值的基本统计量分析(表 1 和图 1) 显示, 285 份多样性自交系关联群体的叶向值在不同 年份呈现广泛的连续变异。2018 年叶向值群体均值 高于 2017 年, 表明关联群体总体上在 2017 年更趋 于平展, 2018 年更趋于紧凑上冲, 而 2 年叶向值 BLUP 居中。不同年份叶向值的峰度和偏度的绝对 值都小于 1 (表 1)，表明叶向值在不同年份均服从正 态分布, 说明叶向值为典型的数量性状，受多基因 控制。

方差分析结果表明(表 2), 不同年份关联群体叶 向值的基因型效应、年份效应和基因型 $\times$ 年份互作效 应达到极显著水平 $(P<0.01)$, 而重复间方差不显著 $(P>0.05)$ 。因此，各自交系间的叶向值存在极显著差

表 1 不同年份叶向值的基本统计量分析及相关分析

Table 1 Basic stastical analysis and correlation coefficients of leaf orientation value in two years

\begin{tabular}{ccccccc}
\hline 年份 & 均值 & 变异范围 & 标准差 & 偏度 & 峰度 & 相关系数 Correlation coefficient \\
\cline { 6 - 8 } Year & Mean & Range & SD & Skewness & Kurtosis & 2017 \\
\hline 2017 & 59.45 & $12.99-86.28$ & 13.77 & -0.58 & 0.13 & \\
2018 & 63.86 & $17.05-90.00$ & 13.11 & -0.79 & 0.59 & $0.75^{* *}$ \\
BLUP & 61.58 & $23.46-82.65$ & 11.14 & -0.63 & 0.30 & $0.95^{* *}$ \\
\hline
\end{tabular}

"和**分别表示在 $P<0.05$ 和 $P<0.01$ 水平显著。BLUP: 最佳线性无偏预测。

${ }^{*}$ and ${ }^{* *}$ indicate significance at $P<0.05$ and $P<0.01$, respectively. BLUP: Best Linear Unbiased Prediction.

表 2 不同年份叶向值的方差分析

Table 2 Analysis of variance (ANOVA) for leaf orientation value in two years

\begin{tabular}{lcc}
\hline \multicolumn{1}{c}{ 变异来源 } & 方差分量 & 遗传力 \\
$H^{2}(\%)$
\end{tabular}

“和"*分别表示在 $P<0.05$ 和 $P<0.01$ 水平显著。

* and ${ }^{* *}$ indicate significance at $P<0.05$ and $P<0.01$, respectively. 


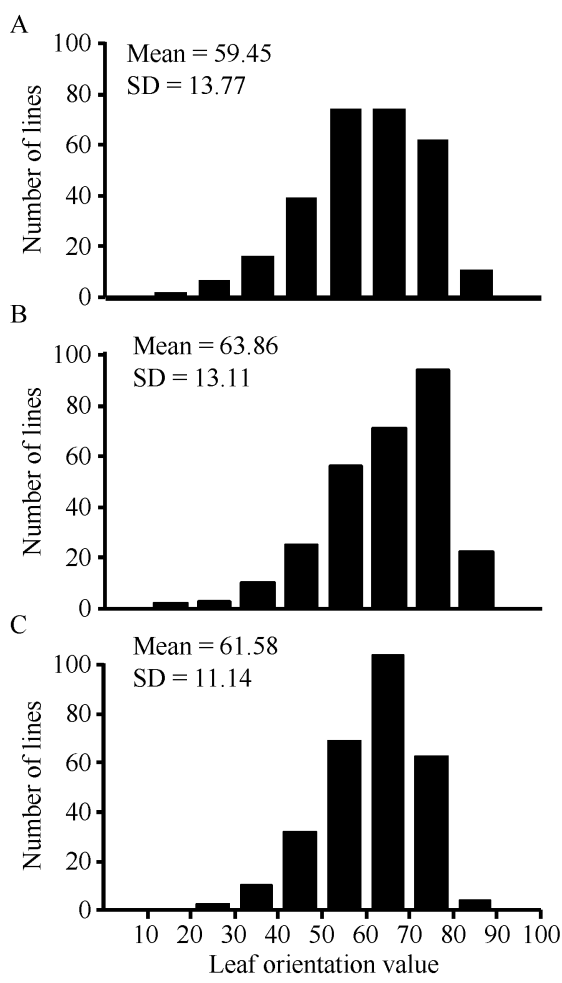

图 1 不同年份玉米叶向值的频率分布图

Fig. 1 Frequency distribution of leaf orientation value in two years

A: 2017 年; B: 2018 年; C: 2 年联合的 BLUP 值。

A: Year of 2017; B: Year of 2018; C: BLUP for combined 2 years.

异, 且年份对叶向值也有显著的影响。叶向值在不 同年份间的遗传力达到 $87.86 \%$ ，提示该性状主要受 遗传因素控制，不同年份的环境效应影响较小。相 关分析结果表明不同年份之间叶向值的相关达到极 显著水平 $(P<0.01)$ 。最佳线性无偏预测(Best Linear Unbiased Prediction, 简称BLUP)可以对多环境数据 整合, 消除环境效应, 得到材料稳定遗传的表型。 BLUP与不同年份叶向值相关系数高达 0.90 以上, 进
一步验证了叶向值具有较高的遗传力, 受环境影响 较小。

\section{$2.2 \mathrm{LD}$ 分析}

285 份材料 10 条染色体平均 LD衰减距离为 457 $\mathrm{kb}$ (图2), 其中 1 号到 10 号染色体 $\mathrm{LD}$ 衰减距离分别为 $332 、 299 、 432 、 500 、 382 、 421 、 445 、 500 、 631$ 和 $694 \mathrm{~kb}$ (图2)。

\section{3 叶向值的全基因组关联分析}

\subsection{1 阈值确定利用GEC软件分析 39,827 个高} 质量 SNP 的有效标记数表明, 有效标记数 Ne为 24,703 个, 建议 $P$ 值为 $4.05 \mathrm{E}-5$, 即 $-\lg P$ 为 4.39 。因此, 该值将作为叶向值全基因组关联分析中标记-性状 显著关联的阈值。

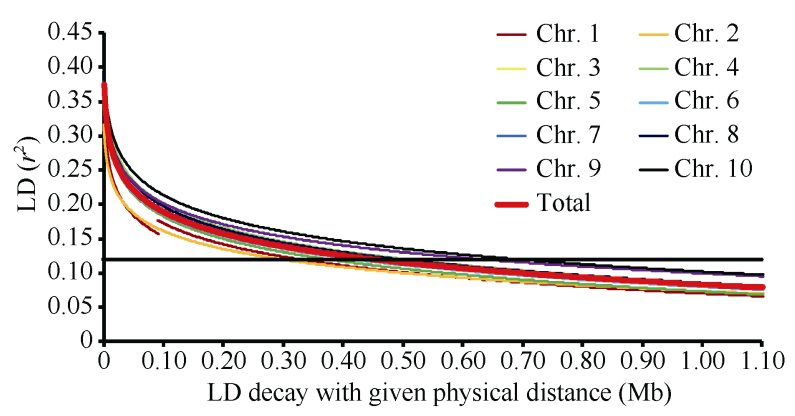

图 2285 份自交系全基因组 LD 衰减图

Fig. 2 Picture of whole-genome LD in the panel of 285 lines

2.3.2 模型选择利用 3 种模型 $(\mathrm{Q} 、 \mathrm{~K}$ 和 $\mathrm{Q}+\mathrm{K})$ 分别 对2个年份叶向值及其BLUP值进行了 GWAS分析。 $\mathrm{QQ}$ 图显示(图3), Q模型(图中绿色点)和 $\mathrm{K}$ 模型(图中 蓝色点)对假阳性的控制效果较差, 而 $\mathrm{Q}+\mathrm{K}$ 模型(图 中紫色点)对假阳性的控制较好。因此，全基因组关 联分析利用基于 $\mathrm{Q}+\mathrm{K}$ 的混合线性模型来检测与叶向 值显著相关的SNP。
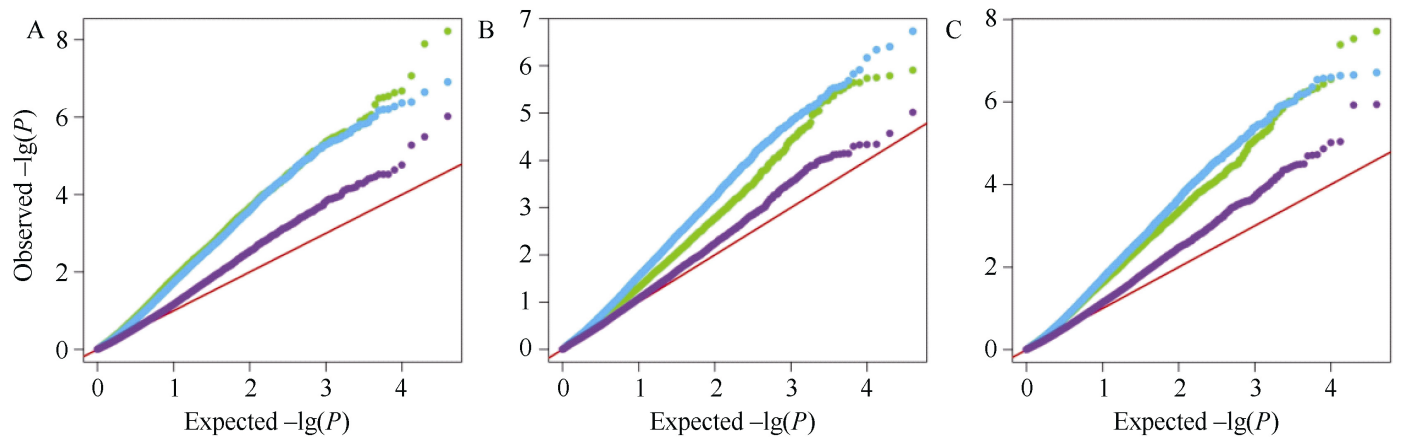

图 3 不同年份玉米叶向值 3 种 GWAS 模型的 QQ 图

Fig. 3 Quantile-quantile (QQ) plots resulting from GWAS results using three methods for leaf orientation value in two years A: 2017 年; B: 2018 年; C: 2 年联合的 BLUP 值。绿色点: Q 模型; 蓝色点: K 模型; 紫色点: Q+K 模型。

A: Year of 2017; B: Year of 2018; C: BLUP for combined two years. Green spot: Q model; blue spot: K model; purple spot: Q+K model. 


\subsection{3 全基因组关联分析利用覆盖基因组的} 39,827 个SNP标记结合285份多样性自交系连续2年 的叶向值表现及其BLUP值进行了全基因组关联分 析。基于 $\mathrm{Q}+\mathrm{K}$ 模型, 一共检测到 27 个与叶向值显著 关联的SNP $(P<4.05 \mathrm{E}-5)$ (图4和表3)。

基于每条染色体的LD衰减距离, 27个与叶向值 显著关联的SNP分布于基因组的 15 个位点, 分别位 于1号、2号、5号、7号和 10 号 5 条染色体上(图5), 其 中 2017 年、2018年和BLUP下分别检测到8个、2个和 12 个位点。
位点1位于 1.02 bin上, 能够在 2017 年和 2 个年份 联合分析(BLUP)下检测到，可以解释 $8.73 \%$ 和 $7.95 \%$ 的表型变异。位点 $2 、 3$ 和 4 都位于 $1.07 \mathrm{bin}$ 上, 其中位 点2能够在 2017 年和 2018 年 2 个环境以及 2 环境联合 (BLUP)均被检测到，且发现7个与叶向值显著关联 的SNP, 可以解释 $6.24 \% \sim 8.49 \%$ 的表型变异; 位点 3 能够在 2017 年和 2 个年份联合分析(BLUP)下检测到, 可以解释 $7.30 \%$ 和 $7.55 \%$ 的表型变异; 位点4能够在 2017年、2018年和 2 环境联合(BLUP)检测到，与叶向 值显著关联的SNP都为PZE-101166565，可以解释

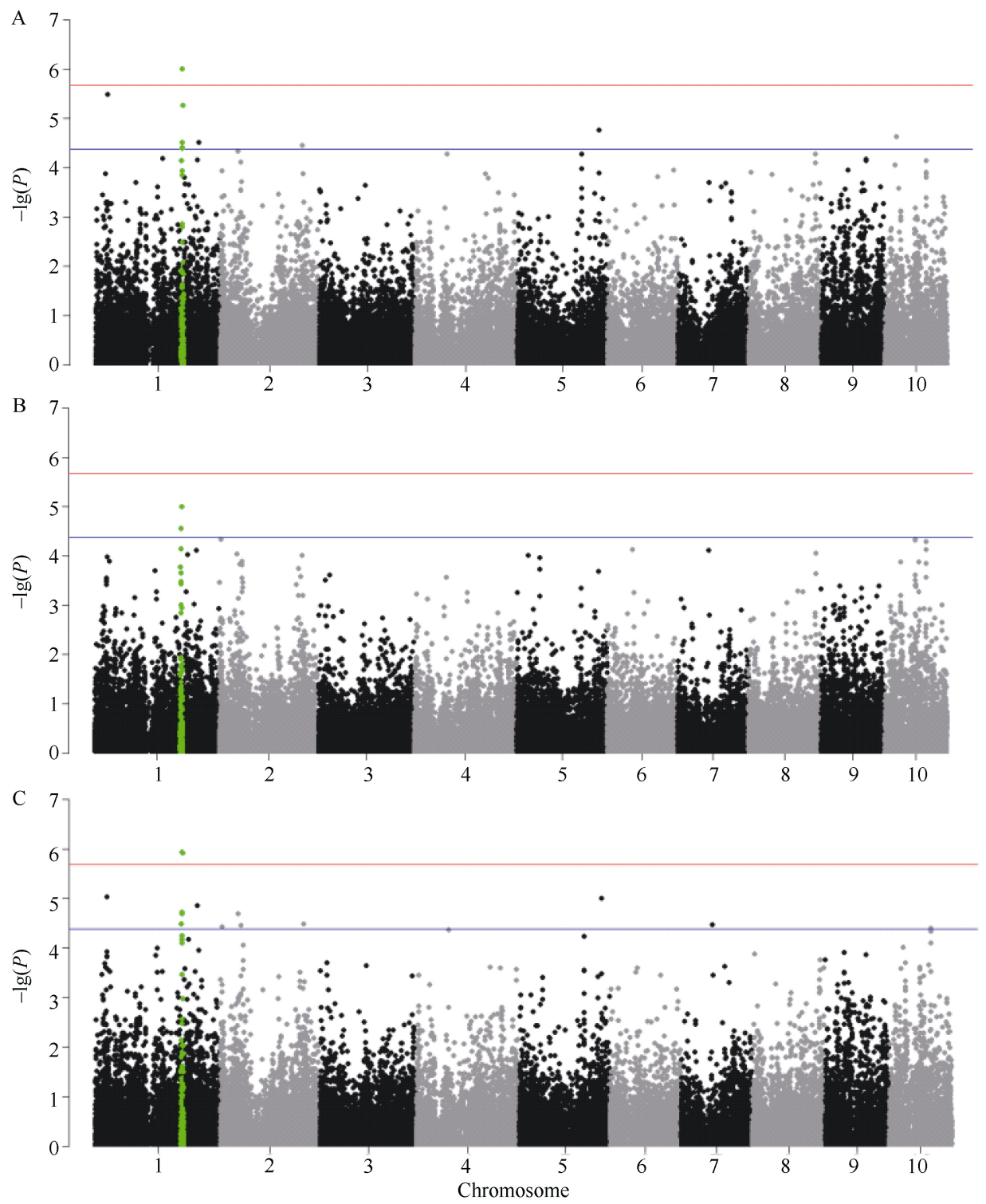

图 4 不同环境下玉米叶向值 $\operatorname{GWAS}(\mathrm{Q}+\mathrm{K}$ 模型)的曼哈顿图

Fig. 4 Manhattan plots resulting from GWAS results using $Q+K$ methods for leaf orientation value in two years A: 2017 年; B: 2018 年; C: 2 年联合的 BLUP 值。绿色点: 位点 2 峰值 SNP 上下游各 50 个 SNP 在不同年份的曼哈顿图。 A: Year of 2017; B: Year of 2018; C: BLUP for combined two years. Green spots: the Manhattan plots of 50 SNPs in the upstream and downstream of lead SNP at site 2 in two years. 


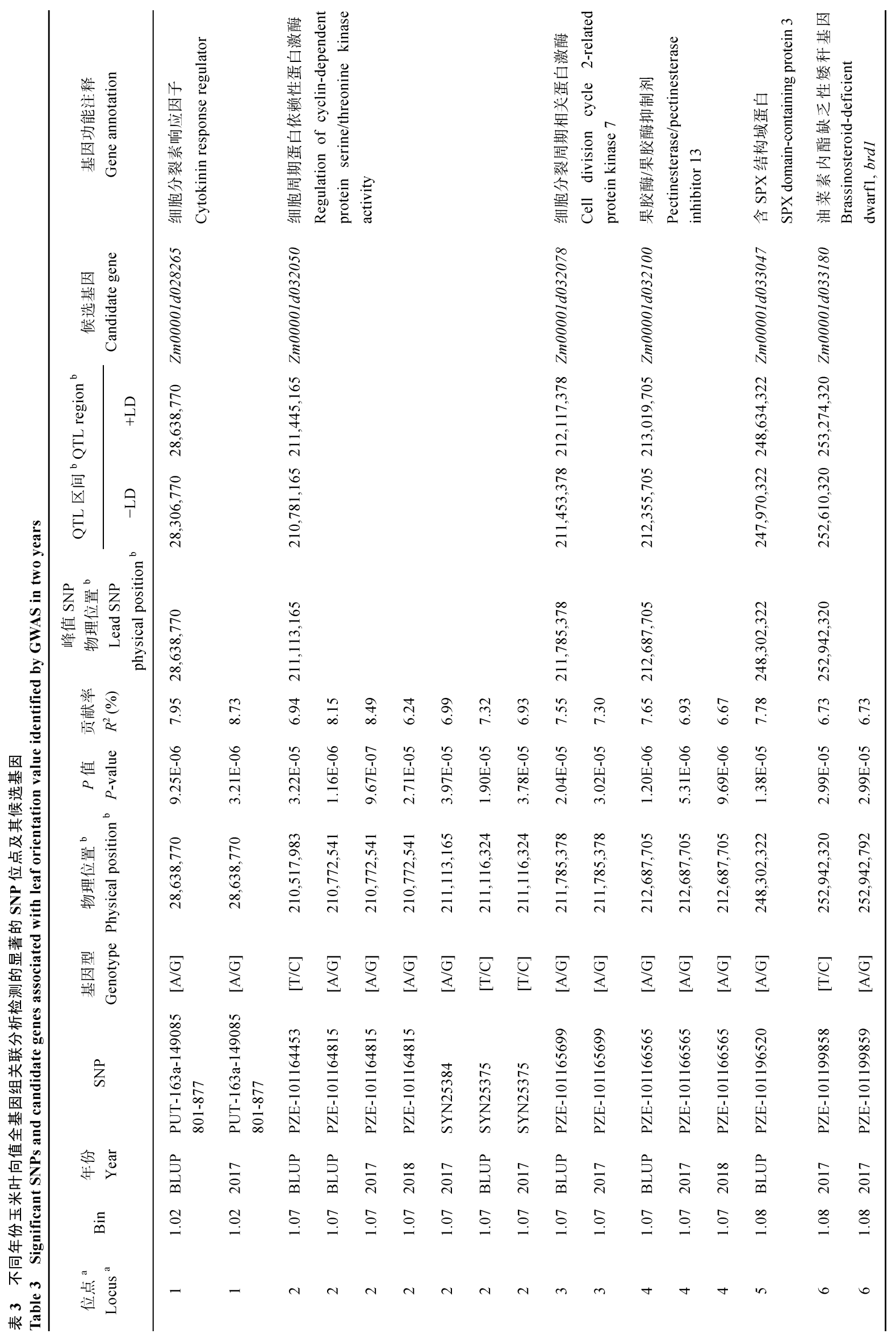




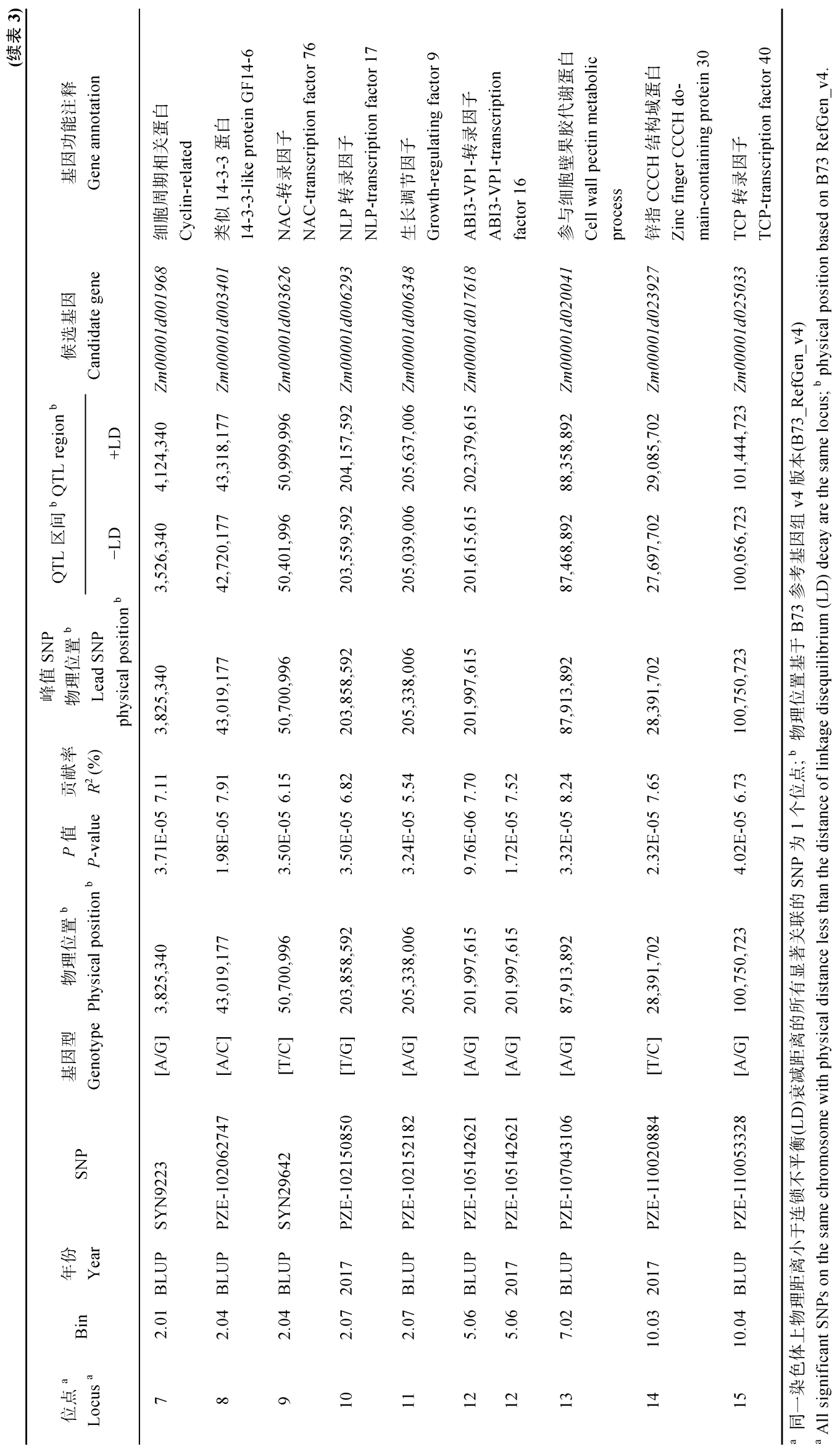




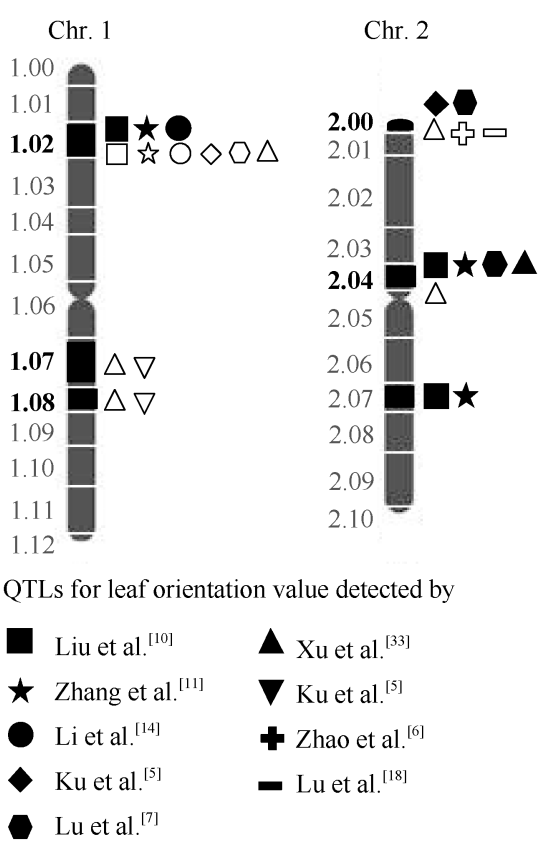

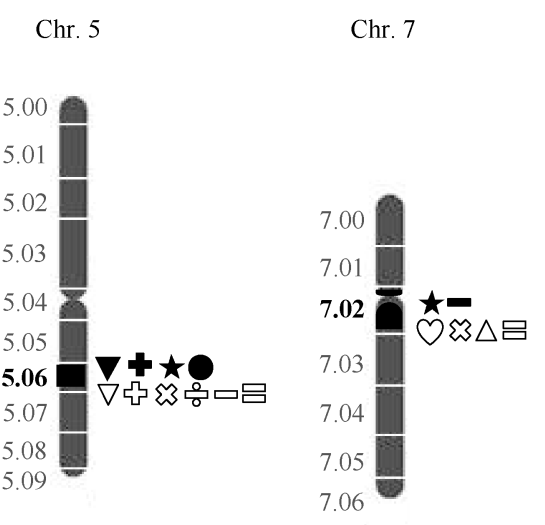

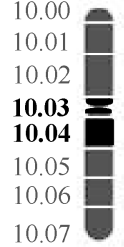

图 5 本研究检测的与叶向值显著关联的位点在染色体上的分布及与前人研究结果的比较

Fig. 5 Distribution of the loci significantly association with leaf orientation value detected in this study on chromosomes and the comparison with the results of previous studies

染色体上粗体的 bin 代表本研究检测的与叶向值显著关联的位点。

Bin with bold on the chromosomes represents the site detected in this study that is significantly association with leaf orientation value.

$6.67 \% \sim 7.65 \%$ 的表型变异。位点 $5 、 6 、 7 、 8 、 9 、 10$ 、 $11 、 13 、 14$ 和 15 , 分布在 1.08 bin、 2.01 bin、 2.04 bin、 $2.07 \mathrm{bin} 、 7.02 \mathrm{bin} 、 10.03 \mathrm{bin}$ 和 $10.04 \mathrm{bin}$ 上, 仅在 1 个环境或者 2 环境联合(BLUP)下检测到, 可解释 $5.54 \% \sim 8.24 \%$ 的表型变异。位点12位于5.06 bin上, 能 够在 2017 年和 2 个年份联合分析(BLUP)下检测到, 可 以解释 $7.52 \%$ 和 $7.70 \%$ 的表型变异。

\subsection{4 叶向值候选基因挖掘基于该群体每条染色}

体的LD衰减距离, 在每个位点内 $-\log _{10}(P)$ 峰值的SNP 上下游各LD衰减距离的区间范围内挖掘候选基因。在 与叶向值显著关联的 15 个位点内共计挖掘 15 个候选基 因 (表 3)。位点 1 至位点 15 的候选基因依次为 Zm00001d028265、Zm00001d032050、Zm00001d032078、 Zm00001d032100、Zm00001d033047、Zm00001d033180、 Zm00001d001968、Zm00001d003401、Zm00001d003626、 Zm00001d006293、Zm00001d006348、Zm00001d017618、 Zm00001d020041、Zm00001d023927和Zm00001d025033。 这些基因主要是与叶枕部位细胞分裂和细胞伸长、植物 激素的生物合成和信号转导、细胞壁的生物合成或降 解或者一些转录因子有关的基因。

\section{3 讨论}

\section{1 叶向值全基因组关联分析结果分析与比较}

根据叶向值的计算公式 ${ }^{[21]}$ 可知, 叶向值是反 映叶片“直”和“立”两个特性的综合指标, 叶夹角越 小(“立”), 叶片越直而不弯(“直”), 叶向值越大。前 人研究也表明, 叶向值与叶夹角极显著负相关(相 关系数为 -0.72 至 -0.81$)$, 但是与叶长和叶宽相关性 不显著 ${ }^{[9,14]}$ 。Zhao等 ${ }^{[6]}{ }^{[} \mathrm{l}^{2}$ 现14个具有叶向值QTL效应的 $\mathrm{mQTL}$ 中, 约 $78.60 \%$ 具有叶夹角QTL效应。因此, 作为 叶向值构成因素之一的叶夹角, 其发育调控的关键基 因也可能对叶向值的形态建成起重要作用 ${ }^{[27]}$ 。

本研究共检测到 15 个与叶向值显著关联的位点, 这 些位点可能是玉米叶向值发育调控的重点区域。其 中, 位点 2 能够在 2017 年和 2018 年 2 个年份环境, 以 及2年份联合的BLUP中均被检测到(图4), 且包含最 多显著关联的 SNP, 效应最大的SNP解释表型变异 超过 $8.00 \%$, 表明这个位点遗传效应较大, 受环境影 响较小, 是叶向值分子设计育种或者叶向值QTL图 位克隆工作重点关注的位点。 
由于前人检测的叶向值QTL主要是采用低密度 的SSR分子标记和基因重组次数有限的连锁作图群 体(如 $\mathrm{F}_{2: 3}$ 或RILs群体), QTL定位的精度较低。因此, 在与前人研究结果相比较时, 若QTL位于相同bin, 则认为不同研究检测的QTL是同一位点(图5) ${ }^{[28]}$ 。

位于 $1.02 \mathrm{bin}$ 上的位点 1 , 在 2017 年解释叶向值 表型变异超过 $8.00 \%$, 遗传效应较大。前人利用不同 群体通过连锁分析在该位点检测到控制叶向值的 QTL ${ }^{[10-11,14]}$ 和叶夹角的QTL ${ }^{[9,17,29-32]}$ 。其中, 张姿丽 等 ${ }^{[11]}$ 同时在3个环境在该位点都检测到叶向值主效 QTL，可解释 $10.10 \%$ 19.90\%的表型变异。于永涛


主效QTL，解释 $14.00 \%$ 26.99\%的表型变异。 $\mathrm{Ku}$ 等 ${ }^{[9]}$ 利用豫 82 和沈 137 构建的 229 个 $\mathrm{F}_{2: 3}$ 家系群体, 通过3 个环境下联合连锁分析, 在 1.02 bin位点上同时检测 到控制叶夹角和叶向值的主效QTL, LOD值分别为 10.8 和 11.0 , 分别解释 $20.40 \%$ 和 $23.20 \%$ 的表型变异。 $\mathrm{Ku}$ 等 ${ }^{[5]}$ 和Zhao 等 ${ }^{[6]}$ 整合前人研究也发现该位点同时 存在叶向值和叶夹角的QTL。因此, 该位点可能存在 1 个或几个不同环境不同遗传背景稳定表达的、同时 控制叶夹角和叶向值的主效基因, 是玉米叶片形态 建成的重要位点, 可用于玉米株型分子育种的前景 标记选择。

位点 $2 、 3 、 4$ 均位于 $1.07 \mathrm{bin}$ 上, 其中位点 2 是本 研究重点发现的位点。利用不同遗传群体, Zhao 等 ${ }^{[6]}$ 和Ding 等 ${ }^{[32]}$ 分别都在 2 个环境下在该位点检测到叶 夹角QTL, 解释 $4.88 \%$ 6.97\%表型变异。然而, 可能 由于群体类型、标记密度或是叶向值QTL定位研究 相对较少, 目前还未发现该位点在调控叶向值中的 重要作用。本研究发现了 3 个位点共计 12 个与叶向值 显著相关的SNP标记都位于1.07 bin上，提示该区段 可能至少有 3 个重要基因参与叶向值的发育调控。

位点 5 和位点 6 都位于 $1.08 \mathrm{bin}$ 上, 包含 3 个与叶 向值显著关联的SNP标记, 在该位点发现的叶向值 和叶夹角QTL较少。Zhao等 ${ }^{[6]}$ 在不同水分处理条件 下，在该位点发现了稳定遗传的叶夹角 QTL，可解 释 $5.30 \%$ 20.58\%的表型变异。Ding等 ${ }^{[32]}$ 也在该位点 发现叶夹角 $\mathrm{QTL}$ 。因此, 该位点的遗传效应, 还需要 更多研究来证实。

位于 $2.01 \mathrm{bin}$ 上的位点 7 , 在 2 年联合的BLUP下 被检测到, 可解释 $7.11 \%$ 的叶向值表型变异。前人利 用不同群体在不同环境下在该位点都检测到了叶向 值QTL ${ }^{[5,7]}$ 和叶夹角 $\mathrm{QTL}^{[7,14,32]}$ 。其中, $\mathrm{Ku}$ 等 ${ }^{[5]}$ 和Zhao
等 ${ }^{[6]}$ 分别整合前人关于叶夹角和叶向值QTL定位结 果, 通过元分析发现 2.01 bin位点存在同时调控叶向 值和叶夹角的 $m Q T L$ 。

位点 8 和位点 9 都位于 $2.04 \mathrm{bin}$ 上, 都是在 2 年联 合的BLUP下发现的，可解释 $6.15 \%$ 7.91\%的叶向值 表型变异。路明等 ${ }^{[7]}$ 、徐德林等 ${ }^{[33]}$ 、刘鹏飞等 ${ }^{[10]}$ 和 张姿丽等 ${ }^{[11]}$ 在该位点均检测到叶向值QTL, 分别解 释 $10.92 \% 、 5.67 \% 、 10.60 \%$ 和 $8.90 \%$ 的表型变异。Ding 等 ${ }^{[32]}$ 还在该位点检测到叶夹角 QTL, 解释 $4.54 \%$ 表 型变异。因此, 该位点可能存在调控玉米叶向值的 主效基因。

2.07 bin上挖掘到位点 10 和位点 11 共 2 个控制叶 向值的位点, 可解释 $5.54 \% \sim 6.82 \%$ 表型变异。刘鹏飞 等 ${ }^{[10]}$ 和张姿丽等 ${ }^{[11]}$ 分别在该位点检测到叶向值QTL, 解释 $6.90 \%$ 和 $5.40 \%$ 的表型变异。总体来看, QTL解释 的表型变异都较低。因此, 该位点可能存在效应较 小的叶向值调控基因。

位于5.06 bin的位点 12 附近检测到多个叶向值

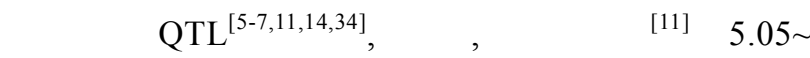
$5.07 \mathrm{bin}$ 上检测到的叶向值QTL可解释 $7.80 \%$ 7.90\% 表型变异, 叶夹角QTL解释 $9.30 \%$ 9.40\%表型变异。 $\mathrm{Ku}$ 等 ${ }^{[5]}$ 在 5.06 检测到 1 个 $\mathrm{mQTL}$ ，同时包含叶夹角 QTL和叶向值QTL。Zhao等 ${ }^{[6]}$ 在5.05 5.06 bin上同样 检测到环境稳定遗传的叶向值QTL和叶夹角 QTL, 可解释 $3.38 \%$ $8.99 \%$ 的表型变异。该位点还需进一步 精细定位, 挖掘其潜在的候选基因。

位点13位于7.02 bin上, 只在2年联合的BLUP下 检测到, 解释叶向值表型变异较高 $(8.24 \%)$ 。在该位 点上，张姿丽等 ${ }^{[11]}$ 检测到 1 个不同环境稳定遗传的 叶向值主效QTL，可解释 $16.40 \%$ 20.10\%的表型变 异; Lu等 ${ }^{[18]}$ 通过GWAS方法分别检测到1个叶向值和 1 个叶夹角 QTL。此外, 一些研究 ${ }^{[5,32,34]}$ 也在该位点检 测到叶夹角 $\mathrm{QTL}$ 。因此, 该基因组区段可能是玉米叶 型性状重要的调控区段。

位点14和位点15分别位于 $10.03 \mathrm{bin}$ 和 $10.04 \mathrm{bin}$, 分别在 2017 年和 2 年联合的BLUP中被检测到。前人 尚未在这两个染色体区段检测到调控叶向值和叶夹 角的QTL。

\section{2 叶向值候选基因挖掘}

叶的发育是植物形态建成的一个重要部分, 其 调控机理十分复杂。叶原基形成后, 在一系列基因 精确调控下, 叶原基开始建立近-远轴(面向茎的为 近轴面, 背向茎的为远轴面)、基-顶轴(由叶的基部 
指向尖部)和中-边轴(从叶的主脉指向边缘)三维轴 向上的极性 ${ }^{[35]}$, 引导原基细胞朝着特定的方向分裂 和分化, 最终发育成一定形态和大小的叶片。叶向 值的形成与基-顶轴和近-远轴极性的建立有关。

根据与叶向值显著关联的峰值 SNP物理位置及 $\mathrm{LD}$ 衰减距离, 从15个位点共计挖掘最有可能的候 选基因 15 个(表 3 )。与前人探索的叶夹角分子调控机 制类似, 叶向值候选基因主要与叶枕部位细胞分裂 和细胞伸长、植物激素的生物合成和信号转导有 关 ${ }^{[2,12-13,16,36-37]}$ 。

植物激素的生物合成和信号转导途径是叶片发 育调控重要分子机制之一。位点 1 的候选基因 Zm00001d028265 (cytokinin response regulator8, crr8), 其功能注释与细胞分裂素信号转导有关。细 胞分裂素是植物生长所必需的激素, 主要作用是调 节细胞增殖 ${ }^{[38]}$ 。因此，推测Zm00001d028265基因可 能通过影响细胞分裂素的信号转导来调控叶枕部位 细胞分裂或者叶片近-远轴面厚壁组织细胞不对称 发育等途径实现对叶向值的调控。此外, 1.02 bin上 的Zm00001d028216基因(indeterminate floral apex1, ifal) 编码 $\mathrm{C} 2 \mathrm{C} 2-\mathrm{YABBY}$ 类型转录因子, 突变体叶片 不能形成正常的中脉, 叶夹角减小, 叶片稍短, 叶 片较窄, 叶鞘较长, 表现披叶的特性 ${ }^{[39]}$ 。ifa 1 与本研 究位点 1 峰值SNP距离 $1.86 \mathrm{Mb}$ 。因此, $1.02 \mathrm{bin}$ 上可能 存在多个控制叶向值的主效基因。位点6的候选基因 Zm00001d033180是参与油菜素内酯(BR)合成途径 的brd1 (brassinosteroid-deficient dwarf1)基因, 距离 本研究峰值SNP仅 $220 \mathrm{~kb}$, 该QTL已被Tian等 ${ }^{[2]}$ 图位 克隆。 $b r d l$ 通过影响叶枕内源 $\mathrm{BR}$ 水平控制叶枕细胞 增殖, 最终影响叶夹角的大小。进一步证实了本研究 所用关联群体通过GWAS手段可有效挖掘调控叶向 值的基因资源。位点5候选基因Zm00001d033047编码 一种SPX结构域蛋白, 参与细胞对磷胁迫和冷胁迫 响应的生物过程。Ruan等 ${ }^{[40]}$ 研究表明, 水稻磷饥饿 诱导蛋白SPX1(Syg1/Pho81/XPR1)和SPX2负调控叶 片倾斜角度。磷缺乏诱导产生SPX1蛋白, 后者与叶 倾角调节因子 $1(R L I 1)$ 直接相互作用, 阻止 $R L I 1$ 与下 游基因 BRASSINOSTEROID UPREGULATED1 $(B U 1)$ 和BU1-LIKE 1 COMPLEX1启动子结合, 从而 抑制水稻叶枕细胞伸长, 限制叶枕的大小, 诱导叶 片直立。位点 8 候选基因 Zm00001d003401编码类似 14-3-3蛋白, 是植物初级代谢和细胞信号转导的主 要调节因子, 参与油菜素内酯的信号转导 ${ }^{[41]}$ 。位点
11 候选基因Zm00001d006348, 编码生长调节因子 (GRF-transcription factor 2, GRF2), 是一种转录激活 因子, 通过控制细胞增殖来调节分生组织功能 ${ }^{[42]}$ 。 在拟南芥叶片形态建成中, 由 $m i R 396$ 介导的生长调 节因子(AtGRFs)调控叶片近-远轴向的细胞极性分 化 ${ }^{[42]}$ 。位点12候选基因Zm00001d017618编码一种B3 转录因子, 调节BR合成途径和信号转导途径基因的 表达 ${ }^{[2,43]}$ 。最近 Tian等 ${ }^{[2]}$ 发现玉米2号染色体上的B3 转录因子Zm00001d002562 (ABI3-VP1-transcription factor 12)基因(即 $Z m R A V L 1$ )激活下游基因 $b r d 1$ 的表 达, 从而提高叶枕内源BR水平, 影响叶枕细胞的增 殖, 导致叶夹角增大。位点 14 候选基因 Zm00001 $d 023927$ 编码一种含锌指 $\mathrm{CCCH}$ 结构域的蛋白质。 Wang 等 ${ }^{[44]}$ 研究表明, 水稻中 $O s L I C$ 基因编码一种锌 指 $\mathrm{CCCH}$ 结构域蛋白, 负调控BR信号。抑制内源 $O s L I C$ 基因表达, 会导致水稻叶片和分菜角显著增 加, 株高降低, 从而降低产量。

叶枕部位近轴面和远轴面细胞分裂的不平衡也 可调控叶向值。位点 2 是本研究发现的最重要位点, 其候选基因 Zm00001d032050功能注释具有细胞周 期蛋白依赖性蛋白激酶 (cyclin-dependent protein kinase, CDK)活性。该蛋白激酶是细胞周期调控机制 的核心, 细胞周期蛋白(cyclin)依次激活相应的细胞 周期蛋白依赖性激酶(CDK), 推动细胞跨越细胞周 期各时相转换的限制点, 是推动细胞周期运行的动 力因素 ${ }^{[45]}$ 。其水稻同源基因 CycD2 (Os06g0217900) 是一种与Cyclin-D3-1类似的细胞周期蛋白。水稻细 胞周期蛋白可通过控制远轴面厚壁组织细胞增殖, 正向调控叶片的直立性, 已被前人证实 ${ }^{[46]}$ 。因此, 有 必要进一步精细定位并图位克隆该位点的功能基 因。位点3的候选基因Zm00001d032078, 同样具有调 节细胞分裂和细胞周期的蛋白酶活性。其水稻同源 基因 CDKC (Os08g0453800)也是一种细胞周期蛋白 依赖性蛋白激酶。位点 7 候选基因为 $Z m 00001$ $d 001968$ 编码一种细胞周期类似蛋白。位点 10 候选基 因Zm00001d006293编码NLP转录因子。有研究表明, 在缺氮条件下类似NIN蛋白转录因子(NLP转录因子) 与Teostine branched $1 /$ cycloidea/proliferating cell factor (TCP20)相互作用, 调控细胞周期蛋白基因 $C Y C B 1 ; 1^{[47]}$ 。位点 15 候选基因 Zm00001d025033编码 一种TCP转录因子。TCP蛋白是植物特有的 bHLH转 录因子。在玉米中有两个 $\mathrm{TCP}$ 家族的基因被较深入 研究。一个是BADl (branch angle deffective 1)编码 
TCP II 类基因，通过促进细胞增殖影响侧枝角度的 出现, 并影响花序结构 ${ }^{[48]}$ 。另一个是玉米驯化基因 TB1 (teosinte branched 1), 可抑制侧枝的生长和发育, $T B 1$ 基因突变体导致玉米侧枝的增加 ${ }^{[49]}$ 。

叶枕部位机械支撑也是调控叶向值的重要机 制。位点4的候选基因Zm00001d032100编码果胶酯 酶, 参与果胶分解代谢过程和细胞壁修饰等生物 过程。果胶是植物细胞壁的重要组成之一, 与植物 组织结构形成等生物过程密切相关。果胶酯酶对 细胞壁的降解 ${ }^{[50]}$ 和果实软化 ${ }^{[51]}$ 具有重要作用。已 有研究发现, 叶枕部位细胞壁机械强度降低, 叶 夹角增大 ${ }^{[52]}$ 。位点13候选基因Zm00001d020041参与 细胞壁果胶代谢生物过程, 可能影响机械强度或者 细胞扩展 ${ }^{[33]}$ 来调控叶向值。

位点9候选基因 Zm00001d003626编码NAC转录因 子, 该类型转录因子对番茄叶发育具有重要作用 ${ }^{[54]}$, 其调控玉米叶向值的机理还需进一步研究。

\section{4 结论}

发现 15 个与叶向值显著关联 $(P<4.05 \mathrm{E}-5)$ 的位 点, 发掘出 15 个候选基因, 其中 $1.07 \mathrm{bin}$ 是最重要 位点, 其候选基因 Zm00001d032050 有待进一步利 用图位克隆技术和 CRISPR/Cas9 基因敲除等技术 验证。

\section{References}

[1] Lambert R J, Johnson R R. Leaf angle, tassel morphology, and the performance of maize hybrids. Crop Sci, 1978, 818: 499-502.

[2] Tian J, Wang C, Xia J, Wu L, Xu G, Wu W, Li D, Qin W, Han X, Chen Q, Jin W, Tian F. Teosinte ligule allele narrows plant architecture and enhances high-density maize yields. Science, 2019, 365: 658-664.

[3] 申卓, 桑立君, 刘丽丽, 徐涛. 紧凑型玉米的增产机制与选育. 种子科技, 2007, (3): 31-33.

Shen Z, Sang L J, Liu L L, Xu T. Yield increasing mechanism and selection of compact maize. Seed Sci \& Technol, 2007, (3): 31-33 (in Chinese)

[4] Li Y, Ma X, Wang T, Li Y X, Liu C, Liu Z, Sun B, Shi Y, Song Y, Carlone M, Buberk D, Bhardwaj H, Whitaker D, Wilson W, Jones E, Wright K, Sun S, Niebur W, Smith S. Increasing maize productivity in China by planting hybrids with germplasm that responds favorably to higher planting densities. Crop Sci, 2011, 51: 2391-2400.

[5] Ku L X, Zhang J, Guo S L, Liu H Y, Zhao R F, Chen Y H. Integrated multiple population analysis of leaf architecture traits in maize (Zea mays L.). J Exp Bot, 2012, 63: 261-274.

[6] Zhao X, Fang P, Zhang J, Peng Y. QTL mapping for six ear leaf architecture traits under water-stressed and well-watered conditions in maize (Zea mays L.). Plant Breed, 2018, 137: 60-72.
[7] 路明, 周芳, 谢传晓, 李明顺, 徐云碧, Warburton M, 张世煌. 玉米杂交种掖单 13 号的 SSR 连锁图谱构建与叶夹角和叶向值 的 QTL 定位与分析. 遗传, 2007, 29: 1131-1138.

Lu M, Zhou F, Xie C X, Li M S, Xu Y B, Warburton M, Zhang S H. Construction of an SSR linkage map and mapping of quantitative trait loci (QTL) for leaf angle and leaf orientation with an elite maize hybrid. Hereditas (Beijing), 2007, 29: 1131-1138 (in Chinese with English abstract).

[8] 孙海艳, 蔡一林, 王久光, 王国强, 徐德林, 徐延军. 玉米株 型性状的 QTL 定位. 西南大学学报(自然科学版), 2010, 32(12): 14-18.

Sun H Y, Cai Y L, Wang J G, Wang G Q, Xu D L, Xu Y J. QTL Mapping for plant-tape traits in maize. $J$ Southwest Univ (Nat Sci Edn), 2010, 32(12): 14-18 (in Chinese with English abstract).

[9] Ku L X, Zhao W M, Zhang J, Wu L C, Wang C L, Wang P A, Zhang W Q, Chen Y H. Quantitative trait loci mapping of leaf angle and leaf orientation value in maize (Zea mays L.). Theor Appl Genet, 2010, 121: 951-959.

[10] 刘鹏飞, 蒋锋, 王汉宁, 王晓明. 玉米叶夹角和叶向值的 QTL 定位. 核农学报, 2012, 26: 231-237.

Liu P F, Jiang F, Wang H N, Wang X M. QTL mapping for leaf angle and leaf orientation in corn. J Nucl Agric Sci, 2012, 26: 231-237 (in Chinese with English abstract).

[11] 张姿丽, 刘鹏飞, 蒋锋, 陈青春, 张媛, 王晓明, 王汉宁. 基于 四交群体的玉米叶夹角和叶向值 QTL 定位分析. 中国农业大 学学报, 2014, 19(4): 7-16.

Zhang Z L, Liu P F, Jiang F, Chen Q C, Zhang Y, Wang X M, Wang H N. QTL mapping for leaf angle and leaf orientation in maize using a four-way cross population. J China Agric Univ, 2014, 19(4): 7-16 (in Chinese with English abstract).

[12] Zhang J, Ku L X, Han Z P, Guo S L, Liu H J, Zhang Z Z, Cao L $\mathrm{R}$, Cui X J, Chen Y H. The ZmCLA4 gene in the qLA4-1 QTL controls leaf angle in maize (Zea mays L.). J Exp Bot, 2014, 65: 5063-5076.

[13] Ren Z, Wu L, Ku L, Wang H, Zeng H, Su H, Wei L, Dou D, Liu H, Cao Y, Zhang D, Han S, Chen Y. ZmILIl regulates leaf angle by directly affecting liguleless 1 expression in maize. Plant Biotechnol J, 2019: DOI: https://doi.org/10.1111/pbi.13255

[14] Li C, Li Y, Shi Y, Song Y, Zhang D, Buckler E S, Zhang Z, Wang $\mathrm{T}$, Li Y. Genetic control of the leaf angle and leaf orientation value as revealed by Ultra-High density maps in three connected maize populations. PLoS One, 2015, 10: $\mathrm{e} 0121624$.

[15] 王会涛, 柳华峰, 郑耀刚, 赵帅帅, 刘浩浩, 库丽霞, 陈彦惠. 玉米叶型相关性状 QTL 定位及上位性效应分析. 分子植物育 种, 2018, $16: 4955-4963$.

Wang H T, Liu H F, Zheng Y G, Zhao S S, Liu H H, Ku L X, Chen Y H. QTL location and epistatic effect analysis of related traits of leaf type in maize. Mol Plant Breed, 2018, 16: 4955-4963 (in Chinese with English abstract).

[16] Tian F, Bradbury P J, Brown P J, Hung H, Sun Q, Flint-Garcia S, Rocheford T R, McMullen M D, Holland J B, Buckler E S. Genome-wide association study of leaf architecture in the maize nested association mapping population. Nat Genet, 2011, 43: 159-162.

[17] 孙娇, 赵美爱, 潘顺祥, 裴玉贺, 郭新梅, 宋希云. 玉米叶夹 角的全基因组关联分析. 华北农学报, 2018, 33(1): 60-64. 
Sun J, Zhao M A, Pan S X, Pei Y H, Guo X M, Song X Y. Correlation analysis of maize leaf angle with genome-wide association analysis. Acta Agric Boreali-Sin, 2018, 33(1): 60-64 (in Chinese with English abstract).

[18] Lu S, Zhang M, Zhang Z, Wang Z, Wu N, Song Y, Wang P. Screening and verification of genes associated with leaf angle and leaf orientation value in inbred maize lines. PLoS One, 2018, 13: e0208386.

[19] Li Y, Shi Y S, Cao Y S, Wang T Y. Establishment of a core collection for maize germplasm preserved in Chinese National GenBank using geographic distribution and characterization data. Genet Resour Crop Evol, 2004, 51: 845-852.

[20] Wu X, Li Y, Li X, Li C, Shi Y, Song Y, Zheng Z, Li Y, Wang T. Analysis of genetic differentiation and genomic variation to reveal potential regions of importance during maize improvement. BMC Plant Biol, 2015, 15: 256.

[21] Pepper G E, Pearce R B, Mock J J. Leaf orientation and yield of maize. Crop Sci, 1977, 17: 883-886.

[22] Purcell S, Neale B, Todd-Brown K, Thomas L, Ferreira M, Bender D, Maller J, Sklar P, de Bakker P I, Daly M J, Sham P C. PLINK: a tool set for whole-genome association and population-based linkage analyses. Am J Hum Genet, 2007, 81: 559-575.

[23] Riedelsheimer C, Czedik-Eysenberg A, Grieder C, Lisec J, Technow F, Sulpice R, Altmann T, Stitt M, Willmitzer L, Melchinger A E. Genomic and metabolic prediction of complex heterotic traits in hybrid maize. Nat Genet, 2012, 44: 217-220.

[24] Yan J, Shah T, Warburton M L, Buckler E S, McMullen M D, Crouch J. Genetic characterization and linkage disequilibrium estimation of a global maize collection using SNP markers. PLoS One, 2009, 4: e8451.

[25] Li M X, Yeung J M, Cherny S S, Sham P C. Evaluating the efective numbers of independent tests and significant $P$-value thresholds in commercial genotyping arrays and public imputation reference datasets. Hum Genet, 2012, 131: 747-756.

[26] 刘坤, 张雪海, 孙高阳, 问鹏帅, 郭海平, 陈思远, 薛亚东, 郭 战勇, 谢惠玲, 汤继华, 李卫华. 玉米株型相关性状的全基因 组关联分析. 中国农业科学, 2018, 51: 821-834.

Liu K, Zhang X H, Sun G Y, Yan P S, Guo H P, Chen S Y, Xue Y D, Guo Z Y, Xie H L, Tang J H, Li W H. Genome-wide association studies of plant type traits in maize. Sci Agric Sin, 2018, 51: 821-834.

[27] Wang Y, Li H, Zhang L, Lyu W, Wang J. On the use of mathematically-derived traits in QTL Mapping. Mol Breeding, 2012, 29: 661-673.

[28] Schön C C, Dhillon B S, Utz H F, Melchinger A E. High congruency of QTL positions for heterosis of grain yield in three crosses of maize. Theor Appl Genet, 2010, 120: 321-332.

[29] 于永涛, 张吉民, 石云素, 宋燕春, 王天宇, 黎裕. 利用不同 群体对玉米株高和叶片夹角的 QTL 分析. 玉米科学, 2006, 14(2): 88-92.

Yu Y, Zhang J, Shi Y, Song Y, Wang T, Li Y. QTL analysis for plant height and leaf angle by using different populations of maize. J Maize Sci, 2006, 14(2): 88-92 (in Chinese with English abstract).

[30] 刘正, 余婷婷, 梅秀鹏, 陈浙宁, 王国强, 王久光, 刘朝显, 王
旭，蔡一林. 玉米穗上叶夹角和叶间距的 QTL 定位. 农业生 物技术学报, 2014, 22: 177-187.

Liu Z, Yu T T, Mei X P, Chen X N, Wang G Q, Wang J G, Liu C $X$, Wang X, Cai Y L. QTL mapping for leaf angle and leaf space above ear position in maize (Zea mays L.). J Agric Biotech, 2014, 22: 177-187 (in Chinese with English abstract).

[31] Chen X, Xu D, Liu Z, Yu T, Mei X, Cai Y. Identification of QTL for leaf angle and leaf space above ear position across different environments and generations in maize (Zea mays L.). Euphytica, 2015, 204: 395-405.

[32] Ding J, Zhang L, Chen J, Li X, Li Y, Cheng H, Huang R, Zhou B, Li Z, Wang J, Wu J. Genomic dissection of leaf angle in maize (Zea mays L.) using a four-way cross mapping population. PLoS One, 2015, 10: $\mathrm{e} 0141619$.

[33] 徐德林, 蔡一林, 吕学高, 代国丽, 王国强, 王久光, 孙海艳, 覃鸿妮. 玉米株型性状的 QTL 定位. 玉米科学, 2009, 17(6): 27-31.

Xu D L, Cai Y L, Lyu X G, Dai G L, Wang G Q, Wang J G, Sun H Y, Tan H N. QTL mapping for plant-tape traits in maize. J Maize Sci, 2009, 17(6): 27-31 (in Chinese with English abstract).

[34] Mickelson S M, Stuber C S, Senior L, Kaeppler S M. Quantitative trait loci controlling leaf and tassel traits in a $\mathrm{B} 73 \times \mathrm{Mo} 17$ population of maize. Crop Sci, 2002, 42: 1902-1909.

[35] Bowman J L, Eshed Y, Baum S F. Establishment of polarity in angiosperm lateral organs. Trends Genet, 2002, 18: 134-141.

[36] 袁立敏. 玉米叶枕发育及叶夹角形成关键调控基因的挖掘. 山东农业大学硕士学位论文, 山东泰安, 2016.

Yuan L M. Mining Key Genes Involved in the Regulation of Ligular Region Development and Leaf Angle (LA) Formation in Maize. MA Thesis of Shandong Agricultural University, Tai'an, Shandong, China, 2016 (in Chinese with English abstract).

[37] Kong F, Zhang T, Liu J, Heng S, Shi Q, Zhang H, Wang Z, Ge L, Li P, Lu X, Li G. Regulation of leaf angle by auricle development in maize. Mol Plant, 2017, 10: 516-519.

[38] Depuydt S, Hardtke C S. Hormone signaling crosstalk in plant growth regulation. Curr Biol, 2011, 21: 365-373.

[39] Strable J, Wallace J G, Unger-Wallace E, Briggs S, Bradbury P J, Buckler E S, Vollbrechta E. Maize $Y A B B Y$ genes drooping leaf1 and drooping leaf2 regulate plant architecture. Plant Cell, 2017, 29: $1622-1641$

[40] Ruan W, Guo M, Xu L, Wang X, Zhao H, Wang J, Yi K. An SPX-RLI1 module regulates leaf inclination in response to phosphate availability in rice. Plant Cell, 2018, 30: 853-870.

[41] Bai M Y, Zhang L Y, Gampala S S, Zhu S W, Song W Y, Chong K, Wang Z Y. Functions of OsBZR1 and 14-3-3 proteins in Brassinosteroid signaling in rice. Proc Natl Acad Sci USA, 2007, 104: 13839-13844.

[42] Wang L, Gu X, Xu D, Wang W, Wang H, Zeng M, Chang Z, Huang H, Cui X. miR396-targeted AtGRF transcription factors are required for coordination of cell division and differentiation during leaf development in Arabidopsis. J Exp Bot, 2011, 62: 761-773.

[43] Je B I, Piao H L, Park S J, Park S H, Kim C M, Xuan Y H, Park S H, Huang J, Choi Y D, An G, Wong H L, Fujioka S, Kim M C, Shimamoto K, Han C. RAV-Likel maintains Brassinosteroid homeostasis via the coordinated activation of BRII and biosynthetic 
genes in rice. Plant Cell, 2010, 22: 1777-1791.

[44] Wang L, Xu Y, Zhang C, Ma Q, Joo S H, Kim S K, Xu Z, Chong K. OsLIC, a novel CCCH-type zinc finger protein with transcription activation, mediates rice architecture via Brassinosteroids signaling. PLoS One, 2008, 3: e3521.

[45] 高磊, 石有珍, 任玉红, 范瑞文, 王爱荣. CDKs(细胞周期依赖 性蛋白激酶)调控细胞周期中的作用. 畜牧兽医杂志, 2010, 29(2): 41-42.

Gao L, Shi Y Z, Ren Y H, Fan R W, Wang A R. Function of CDKs at cell cycle regulation. J Anim Sci \& Veter Med, 2010, 29(2): 41-42 (in Chinese with English abstract).

[46] Sun S, Chen D, Li X, Qiao S, Shi C, Li C, Shen H, Wang X. Brassinosteroid signaling regulates leaf erectness in Oryza sativa via the control of a specific U-type cyclin and cell proliferation. Dev Cell, 2015, 34: 220-228.

[47] Guan P, Ripoll J J, Wang R, Vuong L, Bailey-Steinitz L J, Ye D, Crawford N M. Interacting TCP and NLP transcription factors control plant responses to nitrate availability. Proc Natl Acad Sci USA, 2017, 114: 2419-2424.

[48] Bai F, Reinheimer R, Durantini D, Kellogg E A, Schmidt R J. TCP transcription factor, BRANCH ANGLE DEFECTIVE 1
$(B A D 1)$, is required for normal tassel branch angle formation in maize. Proc Natl Acad Sci USA, 2012, 109: 12225-12230.

[49] Doebley J, Stec A, Gustus C. Teosinte branched 1 and the origin of maize: Evidence for epistasis and the evolution of dominance. Genetics, 1995, 141: 333-346.

[50] Micheli F. Pectin methylesterases: cell wall enzymes with important roles in plant physiology. Trends Plant Sci, 2001, 6: 414-419.

[51] Brummell D A, Cin V D, Crisosto C H, Labavitch J M. Cell wall metabolism during maturation, ripening and senescence of peach fruit. J Exp Bot, 2004, 55: 2029-2039.

[52] Ning J, Zhang B C, Wang N L, Zhou Y H, Xiong L Z. Increased leaf angle1, a Raf-like MAPKKK that interacts with a nuclear protein family, regulates mechanical tissue formation in the lamina joint of rice. Plant Cell, 2011, 23: 4334-4347.

[53] Wolf S, Greiner S. Growth control by cell wall pectins. Protoplasma, 2012, 249(S2): 169-175.

[54] Berger Y, Harpaz-Saad S, Brand A, Melnik H, Sirding N, A1varez J P, Zinder M, Samach A, Eshed Y, Ori N. The NAC-domain transcription factor GOBLET specifies leaflet boundaries in compound tomato leaves. Development, 2009, 136: 823-832. 